\title{
Modeling for Driver Decision-Making Behavior during Amber Signal Time at Intersection
}

\author{
Qi Weiwei ${ }^{1, a}$, Pei Yulong ${ }^{1, b}$, Song Mo ${ }^{1, c}$ \\ ${ }^{1}$ School of Transportation Science and Engineering, Harbin Institute of Technology, \\ Harbin 150090, China \\ aqwwhit@163.com, byulongp@263.net, 07smhit@163.com
}

Keywords: amber light; red clearance; amber interval dilemma; game theory model; traffic safety

\begin{abstract}
Current traffic lights are composed of red, amber, and green, and the drivers' behavior choices and the content of the relevant laws \& regulations during the amber light are disputed. For current signal intersections, the amber light time and the red clearance aren't differentiate from each other, and most of the time, the amber light time acts as red clearance. It will not improve the traffic operating efficiency of the intersection, but bring more traffic conflicts, if the role of the amber light returns to academic definition, in which the amber is a warning signal. In this paper, the process that the drivers choose stopping by slowing down or passing by maintaining the speed during amber time is analyzed through studying the vehicle dynamic characteristics. The generalized amber interval dilemma is defined to establish the mixed-strategy game theory model, which reveals the utility of the driver and signal manager during the amber interval, and the research in algorithm of Nash Equilibrium point is also a focus. According to Nash Equilibrium, the optimal solution of the game theory model is (acceleration, amber setting) or (deceleration, amber canceling).
\end{abstract}

\section{Introduction}

Method of amber timing plays a significant role in the safety and efficiency of intersections. However, the purpose of amber interval in a signal cycle is not clearly defined in current traffic regulations. The lack of uniform design method for amber light causes lots of confusions in traffic operation and enforcement in China [1]. The driver characteristics include brake-response time for first-to-stop vehicles, deceleration rates for first-to-stop vehicles, distinguishing characteristics and predictions of first-to-stop versus last-to-go events, and distinguishing characteristics and predictions of red-light-running events [2]. Statistical analyses were used to investigate the effects of the time to stop line, gender, age group, and grade on the average deceleration rates. Results demonstrate that male drivers appear to show slightly higher rates of deceleration than female drivers. This difference increases as the trigger time to stop line decreases. Younger drivers (younger than 40 years old) and older drivers (60 years of age or older) exhibit greater deceleration rates when compared with drivers in the age group from 40 to 59 [3]. The models that characterize driver brake perception-reaction time (PRT), brake time, and stop-go decisions at the onset of an amber indication at a high-speed signalized intersection approach [4]. Many scholars devoted themselves to the optimization amber light time, and the results are applied to the field of urban traffic management and control $[5,6]$. Driver characteristics during amber interval are important for aspects such as intersection safety evaluation, signal timing design and so on. Driver behavior model is proposed based on logistic regression method, and the model is tested by receiver operating characteristic curve $[7,8]$.

\section{Amber Interval Dilemma}

The first type of dilemma zone occurs at locations where amber times are not long enough. This causes a situation, in which some drivers can't stop in time for the red indication without uncomfortable braking, but also can't enter the intersection before the red indication without considerable accelerating. The upping condition represents a traditional definition of dilemma zone, 
which referenced by most traffic engineering textbooks and guides. This situation can be improved with amber intervals that are sufficiently long. ITE (Institute of Transportation Engineers) recommends the following equations for timing the amber interval to eliminate the dilemma zone:

The amber lights up, if the driver decides to brake, then relevant Stopping Distance $\left(s_{H}\right)$ will be consist of Response Distance $\left(s_{\mathrm{Re}}\right)$ and Braking Distance $\left(s_{B}\right)$, which is shown as follows [1]:

$$
s_{H}=s_{\mathrm{Re}}+s_{B}
$$

According to dynamic characteristics of the vehicles, design formula for $S_{\mathrm{Re}}$ and $s_{B}$ are shown as follows [1]:

$$
\begin{gathered}
s_{\mathrm{Re}}=v t_{\mathrm{Re}} \\
s_{B}=v^{2} / 2 b_{v}
\end{gathered}
$$

Where, $v$ represents driving speed of the vehicles $/\left(\mathrm{m} \cdot \mathrm{s}^{-1}\right)$;

$b_{v}$ represents braking deceleration of the vehicles $/\left(\mathrm{m} \cdot \mathrm{s}^{-2}\right)$.

The amber lights up, if the vehicle runs at a relatively high speed and has already approached the stopping line, then in premise of keeping previous speed, the driver should take decision to pass through, that is passing through the stopping line before the red light starts. Then the vehicle will steer in a distance of $s_{F}$ at previous speed during the time of amber light, whose formula is as follows [1]:

$$
s_{F}=v t_{A}
$$

Where, $s_{F}$ represents the distance that the vehicle runs at previous speed during the time of amber light/ (m); $t_{A}$ represents the time of amber light/ (s).

Parking curve and passing curve for the vehicle at time of amber light can be pictured separately from formula (1) and formula (4), which is shown in figure 1. The dash area in the figure 1 represents amber interval dilemma. The diver can neither stop at the stopping line safely, nor pass though the stopping line without acceleration at the amber interval dilemma.

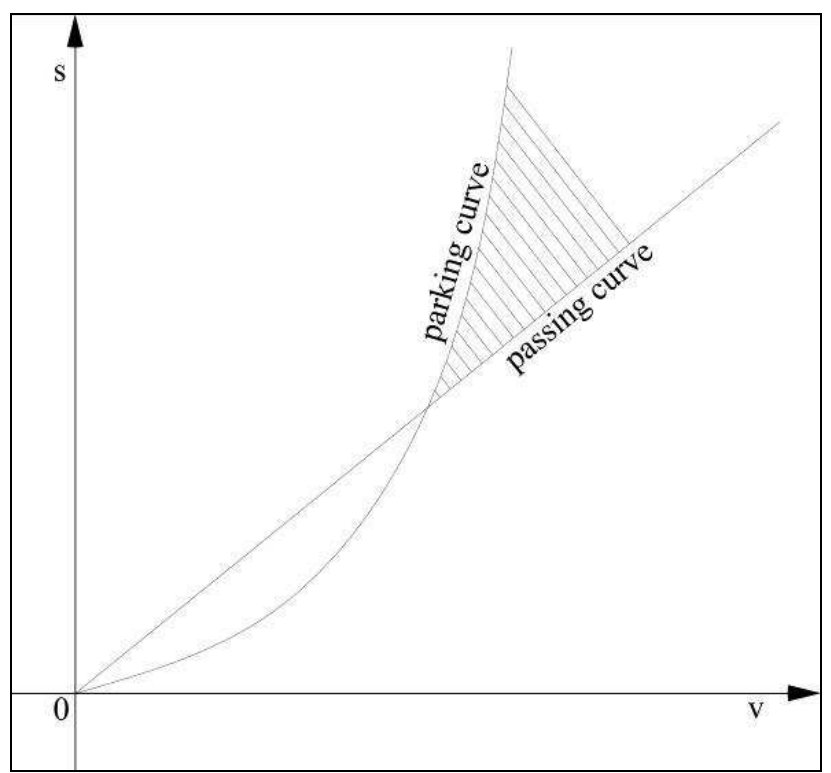

Figure 1 Decision Curve for Drivers

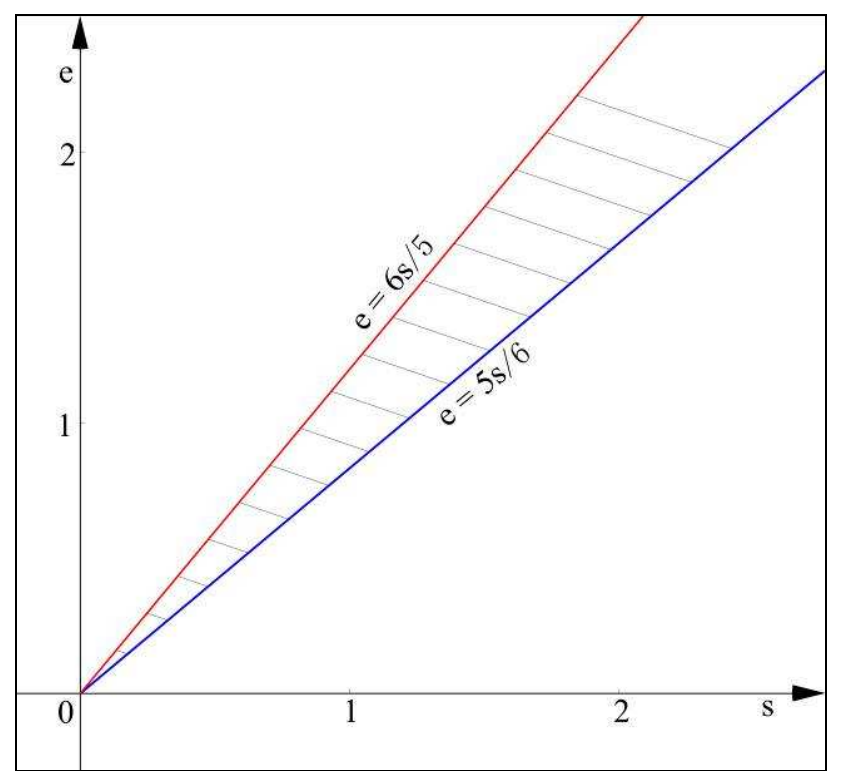

Figure 2 Game Theory Curve for Drivers 
The decision-making behaviors of the drivers in the amber light time of the signal-controlled junctions are established under the driver's absolute obedience to road traffic specification. In reality, the drivers, each of whom has a selfish side, often don't comply with the relevant specifications in amber light period, and to accelerate through the intersection, which leads to serious conflicts. Thus, the next section demonstrates the game theory model between the drivers and managers based on the driver's absolutely rational within the amber light time at signalized intersections.

\section{Game Theory Model}

\section{A. Game Theory Model for Pure Strategy}

The amber light problem is related to traffic efficiency and driving safety, which can evolve into the game theory model between the driver and signal manager.

(1) Assume that the driver only concerns about traffic efficiency, and signal manager only concerns about driving safety in the amber light time, then the game theory model can be established as payoff matrix 1. According to Nash Equilibrium, the optimal solution of the game theory model is (acceleration, amber setting).

\begin{tabular}{|c|c|c|c|}
\hline \multirow{2}{*}{\multicolumn{2}{|c|}{ payoff matrix 1}} & \multicolumn{2}{|c|}{ manager } \\
\hline & & amber setting & amber canceling \\
\hline \multirow{2}{*}{ driver } & acceleration & $u,-u$ & $2 u,-2 u$ \\
\hline & deceleration & $-2 u, 2 u$ & $-u, u$ \\
\hline
\end{tabular}

(2) Assume that the driver only concerns about driving safety, and signal manager only concerns about traffic efficiency in the amber light time, then the game theory model can be established as payoff matrix 2. According to Nash Equilibrium, the optimal solution of the game theory model is (deceleration, amber canceling).

\begin{tabular}{cccc}
\hline & payoff matrix 2 & & manager \\
& & amber setting & amber canceling \\
\hline \multirow{2}{*}{ driver } & acceleration & $-u, u$ & $-2 u, 2 u$ \\
& deceleration & $2 u,-2 u$ & $u,-u$ \\
\hline
\end{tabular}

The analysis result shows that the process of decision-making behavior for the driver and signal manager is concerned about the tendency closely to traffic efficiency or driving safety, which affects the driver's behavior in the amber light significantly. Both traffic efficiency and driving safety should be considered in the research, and a mixed strategy game model will be established to describe the relationship between the driver and signal manager.

B. Game Theory Model for Mixed Strategy

Drivers' selection is single in the matrix 1 and matrix 2, but it is not stationary that drivers select acceleration or deceleration. They select one of the two acts with a certain probability. Assuming $p$ is the probability for drivers to select acceleration, and $(1-p)$ is the probability to select deceleration. Assuming $q$ is the probability for managers to select amber setting, and then $(1-q)$ is the probability to select amber canceling. The game theory model can be established as payoff matrix 3 . The payoff for driver and manager can be expressed as function $\Omega(p, q)$, and according to Nash Equilibrium, the optimal solution of the game theory model is calculated through formula (5) and (6).

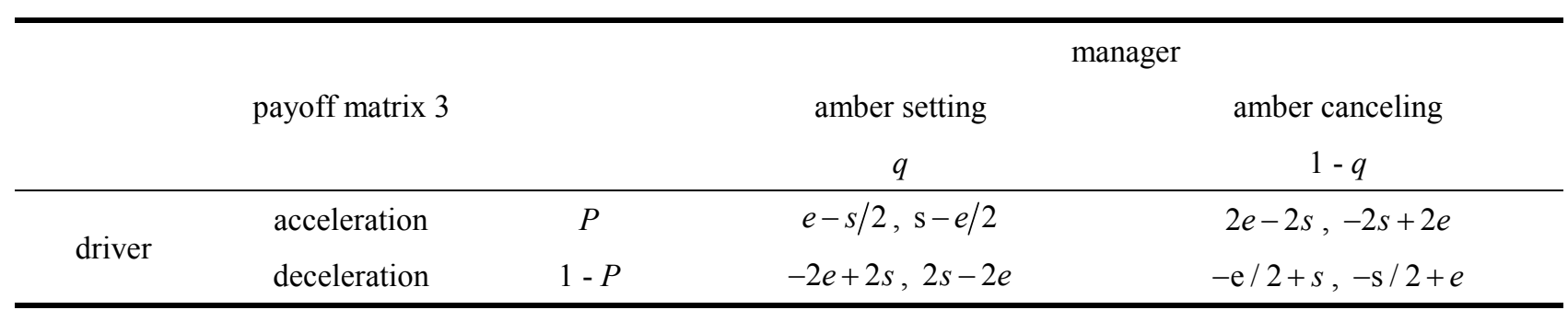




$$
\begin{aligned}
& \Omega_{\text {driver }}(p, q)=p q(e-s / 2)+p(1-q)(2 e-2 s)+(1-p) q(-2 e+2 s)+(1-p)(1-q)(-e / 2+s) \\
& \Omega_{\text {manager }}(p, q)=p q(s-e / 2)+p(1-q)(-2 s+2 e)+(1-p) q(2 s-2 e)+(1-p)(1-q)(-s / 2+e)
\end{aligned}
$$

A first-order differential condition of formula (5) and (6) is as follows:

$$
\left\{\begin{array}{l}
\frac{\partial \Omega_{\text {driver }}}{\partial p}=\left(\frac{1}{2} e+\frac{1}{2} s\right) q+\left(\frac{5}{2} e-3 s\right)=0 \\
\frac{\partial \Omega_{\text {manager }}}{\partial q}=\left(\frac{1}{2} s+\frac{1}{2} e\right) p+\left(\frac{5}{2} s-3 e\right)=0
\end{array}\right.
$$

From formula (7), the Nash Equilibrium $\left(p^{*}, q^{*}\right)$ can be calculated as follows:

$$
\left\{\begin{array}{l}
p^{*}=\frac{3 e-2.5 s}{0.5 e+0.5 s} \\
q^{*}=\frac{3 s-2.5 e}{0.5 s+0.5 e}
\end{array}\right.
$$

Next section, the solution of equation (8) is discussed according to the relation between $e$ (value of traffic efficiency) and $s$ (value of driving safety).

\section{Discussion for Game Theory Model}

The $0 \leq p^{*} \leq 1$ and $0 \leq q^{*} \leq 1$ are the objective conditions of probability function variables, so inequality can be expressed as follows based on the equations (8).

$$
\left\{\begin{array}{l}
0 \leq \frac{3 e-2.5 s}{0.5 e+0.5 s} \leq 1 \\
0 \leq \frac{3 s-2.5 e}{0.5 s+0.5 e} \leq 1
\end{array}\right.
$$

Based on formula (9), it can be calculated as formula (10).

$$
\left\{\begin{array}{l}
\frac{5}{6} s \leq e \leq \frac{6}{5} s \\
\frac{5}{6} e \leq s \leq \frac{6}{5} e
\end{array}\right.
$$

So, Discussion is extended as follows:

1) When $e=\frac{5}{6} s$ and $s=\frac{6}{5} e$, then $p^{*}=0$ and $q^{*}=1$

2) When, $e=\frac{6}{5} s$ and $s=\frac{5}{6} e$, then $p^{*}=1$ and $q^{*}=0$

This inequality (10) can be represented as a shaded area in the two-dimensional axis, which is shown in figure 2. Thus, the condition $e=s$ is a special condition, and the payoff matrix 3 can be updated as payoff matrix 4, in which, we assume $e=s=1$. According to Nash Equilibrium, the optimal solution of the game model is $\left(p^{*}=0.5, q^{*}=0.5\right)$. The results show that drivers will make choice from acceleration or deceleration with probability of 0.5 . 


\begin{tabular}{ccccc}
\hline & & & & manager \\
& payoff matrix 4 & & amber setting & amber canceling \\
& & & $q$ & $1-q$ \\
\hline \multirow{2}{*}{ driver } & acceleration & $P$ & $1 / 2,1 / 2$ & 0,0 \\
& deceleration & $1-P$ & 0,0 & $1 / 2,1 / 2$ \\
\hline
\end{tabular}

\section{Conclusions}

In summary, the optimal solution of the game theory model for pure strategy and mixed strategy is similar, and the following conclusions can be got.

1) The drivers and mangers pay attention to both traffic efficiency and driving safety, and the probability they select which acts is closed with the relation between $e$ and $s$.

2) The drivers who have the selfish side often don't comply with the relevant specifications in amber light period, because that (acceleration, amber setting) is one of the optimal choices in the model for pure strategy.

3) It is rational that the mangers improve traffic operation level by prohibiting passing the stop line in amber light period, because that (deceleration, amber canceling) is one of the optimal choices in the model for pure strategy.

\section{Acknowledgments}

The study was supported by the National Natural Science Foundation of China (51178149).

\section{References}

[1] K. P. Li, P. K. Yang, Y. Ni. Amber Interval Design at Urban Signalized Intersections. Urban Transport of China, July 2010, 8(4): 67-72

[2] T. J. Gates, D. A. Noyce, L. Laracuente, and E. V. Nordheim. Analysis of Driver Behavior in Dilemma Zones at Signalized Intersections. Transportation Research Board of the National Academies, Washington, D. C., 2007, pp. 29-39

[3] I. E. Shawarby, H. Rakha, V. W. Inman, and G. W. Davis. Evaluation of Driver Deceleration Behavior at Signalized Intersections. Transportation Research Board of the National Academies, Washington, D. C., 2007, pp. 29-35

[4] H. Rakha, A. Amer, and I. E. Shawarby. Modeling Driver Behavior within a Signalized Intersection Approach Decision-Dilemma Zone. Transportation Research Board of the National Academies, Washington, D. C., 2008, pp. 16-25

[5] L. G. Zhang, J. D. Fan, J. K. Zhang. The Optimal Model of Chang Interval for the Signalized Intersection. Technology \& Economy in Areas of Communications, 2009, 53(3): 16-18

[6] H. Retzko, M. Boltze. Timing of Inter-green Periods at Signalized Intersections: the German Method. ITE Journal, 1987, 7(1): 23-26

[7] K. J. Long, L. R. He, L. D. Han. Driver Behavior at Signalized Intersection during Yellow Interval. Systems Engineering, 2010, 28(12): 117-120

[8] Papaioannou P. Driver behavior, dilemma zone and safety effects at urban signalized intersections in Greece. Accident Analysis and Prevention, 2007, 39: 147-158 